\title{
Is only fixed positive airway pressure a robust tool for kidney protection in patients with obstructive sleep apnea?
}

\author{
Kyoung Sook Park, Ea Wha Kang \\ Division of nephrology, Department of Internal Medicine; NHIS Ilsan Hospital, Goyang, Korea \\ Correspondence to: Ea Wha Kang, MD, PhD. Division of Nephrology, Department of Internal Medicine, NHIS Ilsan Hospital, 1232 Goyang, Korea. \\ Email: eawha@nhimc.or.kr. \\ Provenance: This is an invited Editorial commissioned by the Section Editor Ning Ding (Department of Respiratory and Critical Care Medicine, The \\ First Affiliated Hospital of Nanjing Medical University, Nanjing, China). \\ Comment on: Marrone O, Cibella F, Pépin JL, et al. Fixed But Not Autoadjusting Positive Airway Pressure Attenuates the Time-dependent Decline in \\ Glomerular Filtration Rate in Patients With OSA. Chest 2018;154:326-34.
}

Submitted Jul 16, 2018. Accepted for publication Sep 28, 2018.

doi: $10.21037 /$ jtd.2018.10.91

View this article at: http://dx.doi.org/10.21037/jtd.2018.10.91

The prevalence of chronic kidney disease (CKD) (1) has increased dramatically in recent years, and is unfortunately expected to continue to increase. Increased CKD prevalence is closely related to rapid increase in hypertension, diabetes, obesity, and metabolic syndrome as well as the aging society $(1,2)$. The public health burden of CKD is not limited to its considerable economic burden for renal replacement therapy but CKD has a major impact on the risk of premature death for cardiovascular diseases (3).

Obstructive sleep apnea (OSA) has also become a global health concern in recent years because of its high prevalence and relevance to a variety of cardiovascular diseases such as hypertension, congestive heart failure, arrhythmia, and sudden cardiac death (4). OSA is reported to be present in $50-90 \%$ of patients with CKD and its high prevalence can be attributed to the overlapping comorbid conditions of both diseases $(5,6)$. Solid epidemiological and experimental evidence has also shown that OSA directly promotes the development and progression of CKD. Nocturnal hypoxia in OSA has a direct effect on kidney damage through tubulointerstitial injury and glomerular hyperfiltration, a correlate of proteinuria and glomerular filtration rate (GFR) decrease owing to the activation of the renin-angiotensin system (RAS) and sympathetic nervous system, endothelial dysfunction, and inflammatory and oxidative status $(5,7)$.

Therefore, considering the link between OSA and CKD, identification of the potential role of OSA treatment on CKD progression is extremely important in both clinical practice and public health policy. The first-line therapy of OSA is the use of continuous positive airway pressure (CPAP) devices. CPAP therapy not only alleviates OSA symptoms and hypoxemia but also ameliorates sympathetic hyperactivity, hypertension, and left ventricular function (8-10), which may translate into therapeutic effects on kidney damage. However, the number of studies on the effect of CPAP treatment on renal function is limited. Recently, a few small sized, short-term follow up studies showed the positive impact of CPAP on renal hyperfiltration and renal function decline in non-CKD and CKD patients (11-14). On the other hand, relatively large long-term studies failed to show a positive effect of CPAP on renal progression $(15,16)$. In a recent randomized controlled trial, 200 moderates to severe OSA patients with cardiovascular disease were randomized to CPAP treatment versus usual care for a median follow-up period of 4.4 years. CPAP treatment did not alter renal function or the occurrence of renal adverse events in this relatively small sized trial (17). We therefore need more compelling evidence because previous studies have not shown consistent results.

A recent publication by Marrone et al. in Chest (18) added important evidence to the literature by evaluating the individual effect of fixed and auto-adjusting CPAP therapy on renal function decline in a relatively large number of patients from the prospective European Sleep Apnea Database (ESADA) cohort. The authors assessed the impact of CPAP on renal function decline during a median of 
541 days in 1,807 samples who had creatinine data at both baseline and follow-up. The study patients were divided into three groups: patients without treatment $(n=144)$, patients on fixed CPAP (fCPAP) $(\mathrm{n}=1,178)$, and patients on auto-adjusting CPAP (APAP) $(\mathrm{n}=485)$. Renal function at baseline was normal or slightly diminished in most patients. The authors reported a novel observation that the rate of renal function decline only decreased by fCPAP and not by APAP in a large, well-characterized cohort of OSA patients for a detailed long-term follow up.

In all study patients, the estimated GFR (eGFR) decreased over time. The strongest reduction in eGFR over time was observed in subjects without treatment, even though OSA severity and degree of comorbidities were lower than those in both CPAP groups. This confirms the independent pathogenic role of OSA in renal function decline and suggests the possible role of the treatment of OSA in renal damage. In addition, better baseline renal function and longer followup duration were significant predictors of a reduction in renal function in a multiple regression analysis. Therefore, from the beginning of OSA diagnosis, significant effort should be made to discover renal damage and maintain renal function. In patients with OSA, impaired renal vasodilating capacity and increased renal vascular resistance and filtration fraction have been reported $(11,12,19)$. Although these conditions transiently preserve renal function, they gradually damage the glomerulus, resulting in the development of glomerulosclerosis. Kinebuchi et al. (11) found that OSA patients were usually in a glomerular-hyperfiltrating status, and that short-term use of CPAP might prevent renal damage by ameliorating glomerular hyperfiltration. Nicholl et al. (12) investigated the effect of CPAP on renal hemodynamics at baseline and in response to angiotensin II, which reflects RAS activity. CPAP therapy was associated with the reduction of glomerular pressure and the down-regulation of renal RAS activity, suggesting a potential pathophysiologic mechanism of renal protection.

Another important research finding by Marrone et al. is the result of comparing the effect of the two modalities of CPAP (fCPAP $v s$. APAP). This study showed that only fCPAP attenuated the progressive reduction of eGFR over time. In addition, while in the untreated and APAP groups, a more marked decrease in eGFR occurred in the subjects with longer follow-up, in the fCPAP group, eGFR remained considerably unchanged regardless of treatment duration. The respective effects of fCPAP and APAP were also affirmed in normotensive subjects, without the confounding effect of antihypertensive agents. So far, no data is available on the possible differences between the two CPAP treatment modalities on renal outcome. The pathophysiological mechanism by which the effects of the two therapies can differ is not exactly known. Meta-analysis and recent randomized trials comparing the treatment effect of fCPAP versus APAP showed comparable effects in the correction of sleep respiratory indices, symptoms, and quality of life $(20,21)$. fCPAP therapy provides continuous fixed pressure at an effective setting confirmed by comprehensive attended polysomnography, which is a standard therapy for OSA. On the other hand, the delivered pressure of APAP devices varies, depending on the airflow, pressure fluctuations, or airway resistance (22). These APAP devices could assist the pressure titration process when the attended CPAP titration cannot be obtained, considering the changes in the pressure requirements (22). Thus, APAP is expected to enhance compliance while reducing the high costs of CPAP titration, and is thus increasingly used for long-term treatment of OSA. However, non-clinically relevant differences were found for CPAP adherence $(20,23)$ and the variations in pressure in APAP may induce microarousals and sleep fragmentation in some patients (24). Persistent higher sympathetic activity and poorer cardiorespiratory coupling were reported in patients who received APAP therapy compared to patients who received fCPAP therapy $(25,26)$. Minimal data is available regarding the role of CPAP treatment modalities on BP reduction, which might explain different effects of CPAP modalities on renal function. Two studies reported a greater reduction in diastolic blood pressure (BP) not by the office BP but by ambulatory BP monitoring after applying fCPAP rather than $\operatorname{APAP}(27,28)$. However, in a recent randomized trial, night-time systolic BP and diastolic BP significantly decreased after 6 months of APAP treatment without modifying daytime and 24-hr BP monitoring. Moreover, the dipper profile was significantly improved by active APAP treatment (29). More definite evidence is needed to explore mechanisms other than the BP lowering effect to demonstrate the superiority of fCPAP on renal function in OSA patients. Several studies have reported the efficacy of conventional CPAP (fCPAP) on reducing inflammation, insulin resistance, and sympathetic activity $(27,30)$. Thus, fCPAP treatment is capable of providing a significant benefit in reducing cardiovascular risk factors, possibly leading to improvement of left ventricular function (8) and reduction of cardiovascular morbidity and mortality (10).

It is also meaningful that CPAP adherence in the samples in this study remained high and comparable in the APAP 
and fCPAP groups $(5.4 \pm 2.1$ vs. $5.3 \pm 2.0 \mathrm{~h} /$ day $)$ during the long-term follow-up duration. CPAP adherence is the most important factor for the effectiveness of CPAP therapy. The large randomized trial showed the significant effect of CPAP on cardiovascular events only for the subgroup of patients with OSA using CPAP for more than $4 \mathrm{~h}$ per day (31). Similar findings were reported in other studies about the positive impact of CPAP on renal function decline in OSA patients. Koga et al. (13) evaluated 38 OSA patients after excluding non-compliant patients with an average CPAP use of $<3.5$ hours/night. Three months after CPAP treatment, significant decreases were observed in the serum creatinine levels and increases were found in the eGFR. Puckrin et al. (14) reported that sufficient use of CPAP (average $>4 \mathrm{~h} /$ night on $>70 \%$ of nights) was significantly associated with a slower rate of eGFR decline and greater reduction of proteinuria in CKD patients after a median follow-up period of 2.3 years. Interestingly, this relationship was found to be independent of baseline renal function and proteinuria. In another prospective observational study, 64 patients with mild to moderate OSA were followed-up after 10 months of CPAP therapy (19). The renal resistive index was decreased only in effectively treated OSA patients [CPAP for $\geq 4 \mathrm{~h}$ per night on average and apneahypopnea index (AHI) was reduced by $\geq 50 \%$ and less than $10 / \mathrm{h}$ ], whereas it remained unchanged when OSA was not effectively treated. Unlike these studies, larger long-term studies did not show renal protective effect of CPAP therapy but lacked data on OSA severity and CPAP adherence in these studies $(15,16)$. The ability to maintain CPAP adherence for a long time can be considered one of the major factors in the positive result of fCPAP in this study. In addition, the better beneficial effect of fCPAP on renal function decline is reemphasized considering similar compliance of the APAP group. The authors revealed two selection biases as limitations of their study. First, of the 1,807 study subjects, only about $10 \%$ of those on the total ESADA registry exhibited a higher rate of comorbidities and more severe OSA than the 17,858 excluded patients in the analysis. Thus, the study results may not be completely applicable for the entire OSA population. Two studies showed a faster decline of renal function in the most severe OSA subgroups with polysomnographic documentation of OSA severity $(32,33)$. The severity of nocturnal hypoxemia was associated with rapid renal function loss by influencing the renal RAS activation in patients with OSA (32). The Standards of Practice Committee of the
American Society of Sleep Medicine recommend that APAP devices are not currently candidates for APAP titration or treatment of moderate to severe OSA patients with significant comorbidities such as CHF, COPD, central sleep apnea syndromes or obesity hypoventilation syndromes. The committee also recommend that patients being treated with fCPAP based on APAP titration or being treated with APAP must have a close clinical follow-up to determine treatment effectiveness and safety (22). Taken together, it is clinically essential to select patients who will respond well to CPAP therapy, while fCPAP might be needed for renal protection for more severe OSA population with comorbidities. Also, since more than $90 \%$ of the subjects were Caucasians, the study results could not be applied to all races.

This report has several drawbacks. First, the standardization of serum creatinine is important for the estimation methods and implementing traceability of the assays, but the measurement techniques were not recorded. Furthermore, data on albuminuria, an early marker of kidney damage, is omitted in this study. Any disturbances in sleep quality such as residual sleep fragmentation or arousal during CPAP therapy were not evaluated to explain the differential impact on renal function of the two CPAP modalities. This suggests the need for new studies on the role of nonhypoxic risk factors for CKD progression in OSA and on their pathophysiological mechanisms.

The study by Marrone et al. (18) extends the findings from previous studies showing significant associations between OSA and kidney damage. Given these findings, it seems prudent to consider screening for early kidney damage in patients with OSA with regular laboratory measurements for renal function (creatinine and eGFR) as well as random urine albumin/creatinine ratios to screen for proteinuria. fCPAP rather than APAP may be used to prevent the development and progression of CKD in the high-risk severe OSA population. In addition, the findings from this large observational study provide a rationale for conclusive randomized clinical trials in determining whether only fCPAP can preserve renal function and improve patient survival. Further randomized clinical trials are also needed to reveal the differences between the two modalities depending on the severity and comorbidities of the OSA.

\section{Acknowledgements}

None. 


\section{Footnote}

Conflicts of Interest: The authors have no conflicts of interest to declare.

\section{References}

1. Coresh J, Selvin E, Stevens LA, et al. Prevalence of chronic kidney disease in the United States. JAMA 2007;298:2038-47.

2. Thomas G, Sehgal AR, Kashyap SR, et al. Metabolic syndrome and kidney disease: a systematic review and meta-analysis. Clin J Am Soc Nephrol 2011;6:2364-73.

3. McCullough K, Sharma P, Ali T, et al. Measuring the population burden of chronic kidney disease: a systematic literature review of the estimated prevalence of impaired kidney function. Nephrol Dial Transplant 2012;27:1812-21.

4. Shamsuzzaman AS, Gersh BJ, Somers VK. Obstructive sleep apnea: implications for cardiac and vascular disease. JAMA 2003;290:1906-14.

5. Turek NF, Ricardo AC, Lash JP. Sleep disturbances as nontraditional risk factors for development and progression of CKD: review of the evidence. Am J Kidney Dis 2012;60:823-33.

6. Lee YC, Hung SY, Wang HK, et al. Sleep apnea and the risk of chronic kidney disease: a nationwide populationbased cohort study. Sleep 2015;38:213-21.

7. Fine LG, Norman JT. Chronic hypoxia as a mechanism of progression of chronic kidney diseases: from hypothesis to novel therapeutics. Kidney Int 2008;74:867-72.

8. Mansfield DR, Gollogly NC, Kaye DM, et al. Controlled trial of continuous positive airway pressure in obstructive sleep apnea and heart failure. Am J Respir Crit Care Med 2004;169:361-6.

9. Gottlieb DJ, Punjabi NM, Mehra R, et al. CPAP versus oxygen in obstructive sleep apnea. N Engl J Med 2014;370:2276-85.

10. Marin JM, Carrizo SJ, Vicente E, et al. Long-term cardiovascular outcomes in men with obstructive sleep apnoea-hypopnoea with or without treatment with continuous positive airway pressure: an observational study. Lancet 2005;365:1046-53.

11. Kinebuchi S, Kazama JJ, Satoh M, et al. Short-term use of continuous positive airway pressure ameliorates glomerular hyperfiltration in patients with obstructive sleep apnoea syndrome. Clin Sci (Lond) 2004;107:317-22.

12. Nicholl DD, Hanly PJ, Poulin MJ, et al. Evaluation of continuous positive airway pressure therapy on renin- angiotensin system activity in obstructive sleep apnea. Am J Respir Crit Care Med 2014;190:572-80.

13. Koga S, Ikeda S, Yasunaga T, et al. Effects of nasal continuous positive airway pressure on the glomerular filtration rate in patients with obstructive sleep apnea syndrome. Intern Med 2013;52:345-9.

14. Puckrin R, Iqbal S, Zidulka A, et al. Renoprotective effects of continuous positive airway pressure in chronic kidney disease patients with sleep apnea. Int Urol Nephrol 2015;47:1839-45.

15. Molnar MZ, Mucsi I, Novak M, et al. Association of incident obstructive sleep apnoea with outcomes in a large cohort of US veterans. Thorax 2015;70:888-95.

16. Chu H, Shih CJ, Ou SM, et al. Association of sleep apnoea with chronic kidney disease in a large cohort from Taiwan. Respirology 2016;21:754-60.

17. Loffler KA, Heeley E, Freed R, et al. Effect of Obstructive Sleep Apnea Treatment on Renal Function in Patients with Cardiovascular Disease. Am J Respir Crit Care Med 2017;196:1456-62.

18. Marrone O, Cibella F, Pepin JL, et al. Fixed But Not Autoadjusting Positive Airway Pressure Attenuates the Time-dependent Decline in Glomerular Filtration Rate in Patients With OSA. Chest 2018;154:326-34.

19. Buchner NJ, Wissing KR, Stegbauer J, et al. The renal resistance index is increased in mild-to-moderate obstructive sleep apnoea and is reduced under continuous positive airway pressure. Nephrol Dial Transplant 2011;26:914-20.

20. Xu T, Li T, Wei D, et al. Effect of automatic versus fixed continuous positive airway pressure for the treatment of obstructive sleep apnea: an up-to-date meta-analysis. Sleep Breath 2012;16:1017-26.

21. Bloch KE, Huber F, Furian M, et al. Autoadjusted versus fixed CPAP for obstructive sleep apnoea: a multicentre, randomised equivalence trial. Thorax 2018;73:174-84.

22. Morgenthaler TI, Aurora RN, Brown T, et al. Practice parameters for the use of autotitrating continuous positive airway pressure devices for titrating pressures and treating adult patients with obstructive sleep apnea syndrome: an update for 2007. An American Academy of Sleep Medicine report. Sleep 2008;31:141-7.

23. Bakker JP, Marshall NS. Flexible pressure delivery modification of continuous positive airway pressure for obstructive sleep apnea does not improve compliance with therapy: systematic review and meta-analysis. Chest 2011;139:1322-30.

24. Marrone O, Insalaco G, Bonsignore MR, et al. Sleep 
structure correlates of continuous positive airway pressure variations during application of an autotitrating continuous positive airway pressure machine in patients with obstructive sleep apnea syndrome. Chest 2002;121:759-67.

25. Patruno V, Tobaldini E, Bianchi AM, et al. Acute effects of autoadjusting and fixed continuous positive airway pressure treatments on cardiorespiratory coupling in obese patients with obstructive sleep apnea. Eur J Intern Med 2014;25:164-8.

26. Karasulu L, Epozturk PO, Sokucu SN, et al. Improving Heart rate variability in sleep apnea patients: differences in treatment with auto-titrating positive airway pressure (APAP) versus conventional CPAP. Lung 2010;188:315-20.

27. Patruno V, Aiolfi S, Costantino G, et al. Fixed and autoadjusting continuous positive airway pressure treatments are not similar in reducing cardiovascular risk factors in patients with obstructive sleep apnea. Chest 2007;131:1393-9.

28. Pepin JL, Tamisier R, Baguet JP, et al. Fixed-pressure CPAP versus auto-adjusting CPAP: comparison of efficacy on blood pressure in obstructive sleep apnoea, a randomised clinical trial. Thorax 2016;71:726-33.

Cite this article as: Park KS, Kang EW. Is only fixed positive airway pressure a robust tool for kidney protection in patients with obstructive sleep apnea? J Thorac Dis 2018;10(Suppl 33):S3819-S3823. doi: 10.21037/jtd.2018.10.91
29. Joyeux-Faure M, Baguet JP, Barone-Rochette G, et al. Continuous Positive Airway Pressure Reduces NightTime Blood Pressure and Heart Rate in Patients With Obstructive Sleep Apnea and Resistant Hypertension: The RHOOSAS Randomized Controlled Trial. Front Neurol 2018;9:318.

30. Harsch IA, Schahin SP, Radespiel-Troger M, et al. Continuous positive airway pressure treatment rapidly improves insulin sensitivity in patients with obstructive sleep apnea syndrome. Am J Respir Crit Care Med 2004;169:156-62.

31. Barbe F, Duran-Cantolla J, Sanchez-de-la-Torre M, et al. Effect of continuous positive airway pressure on the incidence of hypertension and cardiovascular events in nonsleepy patients with obstructive sleep apnea: a randomized controlled trial. JAMA 2012;307:2161-8.

32. Ahmed SB, Ronksley PE, Hemmelgarn BR, et al. Nocturnal hypoxia and loss of kidney function. PLoS One 2011;6:e19029.

33. Jaussent I, Cristol JP, Stengel B, et al. Impact of sleep disturbances on kidney function decline in the elderly. Eur Respir J 2016;47:860-8. 